\title{
Current progress and prospect of immune checkpoint inhibitors in hepatocellular carcinoma (Review)
}

\author{
ZHU ZENG $^{1}$, BIAO YANG ${ }^{2}$ and ZHENG-YIN LIAO ${ }^{1}$ \\ Departments of ${ }^{1}$ Abdominal Oncology and ${ }^{2}$ Gastroenterology, West China Hospital, \\ West China Medical School, Sichuan University, Chengdu, Sichuan 610041, P.R. China
}

Received January 31, 2020; Accepted June 19, 2020

DOI: $10.3892 / \mathrm{ol} .2020 .11909$

\begin{abstract}
In recent years, the incidence of liver cancer has increased and is currently the sixth most common tumor and the second leading cause of cancer-associated mortality worldwide. Most cases of liver cancer are hepatocellular carcinoma (HCC). Surgery, including liver transplantation or resection, and radiofrequency ablation therapies are all considered to be the curative treatment options for early-stage HCC. However, most patients have advanced HCC at the time of diagnosis, contributing to a poor prognosis. Therefore, improved treatment for late-stage HCC is needed. Immune checkpoint inhibitors (ICIs), among which programmed death receptor 1 (PD-1)/PD-ligand 1 and cytotoxic T lymphocyte-associated protein 4 are the representative immunological checkpoints, have shown great promise and progress for HCC treatment.

Correspondence to: Dr Zheng-Yin Liao, Department of Abdominal Oncology, West China Hospital, West China Medical School, Sichuan University, 37 Guoxue Xiang, Chengdu, Sichuan 610041, P.R. China

E-mail: liaozhengyin@163.com
\end{abstract}

Abbreviations: HCC, hepatocellular carcinoma; ICIs, immune checkpoint inhibitors; PD-1, programmed death receptor 1; PD-L1, programmed death ligand 1; CTLA-4, cytotoxic T lymphocyte-associated protein 4; $\mathrm{HBV}$, hepatitis B virus; $\mathrm{HCV}$, hepatitis C virus; BCLC, Barcelona Clinic Liver Cancer; RFA, radiofrequency ablation; TACE, transarterial chemotherapy; TKIs, tyrosine protein kinase; FDA, Food and Drug Administration; TGF- $\beta$, transforming growth factor- $\beta$; TCR, T cell antigen receptor; ASCO, American Society of Clinical Oncology; ESMO, European Society of Oncology; CSCO, Chinese Society of Clinical Oncology; ORR, objective response rate; mOS, median overall survival; SD, stable disease; PR, partial response; DCR, disease control rate; PFS, progression free survival; AEs, adverse events; RCREP, reactive capillary epithelial proliferation; Complete response, CR; VEGF, vascular endothelial growth factor; DEB-TACE, drug-eluting bead transarterial chemoembolization; MMR, mismatch repair; MSI, microsatellite instability

Key words: hepatocellular carcinoma, immune checkpoint inhibitors, PD-1/PD-L1 inhibitors, CTLA-4 inhibitors, biomarkers, locoregional therapy
The present review summarizes recent studies that have focused on ICIs and discusses the present limitations affecting the development of new therapeutic strategies.

\section{Contents}

1. Introduction

2. Immune recognition, tolerance and escape of $\mathrm{HCC}$

3. The role of PD1/PD-L1 in immune tolerance and immune escape of $\mathrm{HCC}$

4. Application of PD-1/PD-L1 inhibitors in HCC

5. The role of CTLA-4 in immune tolerance and immune escape of $\mathrm{HCC}$

6. Applications of CTLA-4 inhibitors in HCC

7. Combination immunotherapy with locoregional therapy in $\mathrm{HCC}$

8. Current concerns

9. Conclusions

\section{Introduction}

According to global cancer reports, there are $\sim 850,000$ new liver cancer cases and 840,000 liver cancer-associated deaths worldwide in 2019 (1,2). The incidence is higher in Asia compared with western countries, especially China, where liver cancer is the sixth most common tumor and the second leading cause of cancer-associated mortality in 2019 (2). Hepatocellular carcinoma (HCC) accounts for $~ 90 \%$ of primary liver cancer and is primarily associated with chronic hepatitis B virus (HBV) (3) and hepatitis C virus (HCV) infection (4), followed by excessive drinking and non-alcoholic fatty-associated liver disease (5). According to the Barcelona Clinic Liver Cancer (BCLC) staging criteria and liver function, liver transplantation or resection are the most common treatments (6). Additionally, radiofrequency ablation (RFA) therapies are feasible for early stage disease. However, unfortunately, $\mathrm{HCC}$ is characterized by a rapid onset and is invasive and fast-growing $(6,7)$. HCC is associated with a high recurrence rate and fatality rate when combined with a history of cirrhosis, which results in the majority of patients losing the opportunity for surgery when considering social or economic factors, such as poor allocation of medical resources and low 
income in deprived region (8). Moreover, the 5-year survival rate is $\sim 12.5 \%$ (8). The development of RFA and transarterial chemotherapy (TACE) offers an additional treatment option for HCC. These local intervention treatments have been widely accepted because several studies have confirmed the potential benefits of combination therapy with targeted agents and cellular immune therapies (9-11). Thus, subsequent studies have demonstrated the efficacy of targeted therapies based on tyrosine protein kinase inhibitors (TKIs) or multikinase inhibitors in HCC, such as sorafenib, lenvatinib, regorafenib and cabozantinib (7,12-14); however, these agents fail to improve survival and so the overall results are still unsatisfactory (15).

The successful application of immunotherapy in malignant melanoma in the US in 2011 (16), as well as its application in other types of cancer, and a deeper understanding of the mechanisms underlying HCC pathogenesis have led researchers to investigate whether immunotherapy could be applied in clinical trials of HCC. Immune checkpoints mainly are comprised of the programmed death receptor 1 (PD-1)/programmed death ligand 1 (PD-L1) and cytotoxic T lymphocyte-associated protein 4 (CTLA-4) (17). To some extent, these checkpoints enhance antitumor immunity as well as exaggerate immune system activation (17). Among the available inhibitors, PD-1 inhibitors are currently thought to be the most promising (18-20). Based on two phase II clinical trials, CheckMate 040 and KEYNOTE-224 in which both nivolumab and pembrolizumab yielded promising results as second line agents after first-line sorafenib treatment $(21,22)$, the Food and Drug Administration (FDA) approved nivolumab in 2017 and pembrolizumab in 2018 as second-line therapies for HCC $(23,24)$. Evidence suggests that compared with the placebo, the PD-1 treatment group had notable potency and improved overall survival (OS) as first-line compared with second-line treatment. However, the latest data from the 2019 American Society of Clinical Oncology (ASCO) were discouraging (25). Overall, PD-1/PD-L1 inhibitors are challenging the role of TKIs as first-line treatments for HCC.

\section{Immune recognition, tolerance and escape of HCC}

Normal liver tissue is exposed to a variety of antigens, including toxins and intestinal microbial products (26-28). Liver cancer always occurs in the context of chronic inflammation, which is an immunosuppressive environment mediated by hepatocytes $(27,29)$. Under these inflammatory conditions, the inhibition of antigen-specific immune monitoring is partially mediated by changes in the molecular expression of immunosuppressive checkpoints and dendritic cell function, increasing regulatory $\mathrm{T}$ cell numbers and the release of immunosuppressive cytokines, such as interleukin-10 (IL-10) and transforming growth factor (TGF)- $\beta$ (30-32). Long-term exposure to antigens can also lead to the overexpression of immunosuppressive checkpoint molecules on $\mathrm{T}$ cells, thus resulting in energy failure or cell exhaustion $(26,31)$. Meanwhile, HCC creates an immunosuppressive microenvironment through the expression of immunosuppressive factors that inhibit antigen presentation and the immune response, thus preventing an effective antitumor response and permitting further escape of immune surveillance $(26,31)$. Through the abnormal expression of antigens, secretion of metabolites and cytokines and the change of the immune microenvironment, liver cancer cells can escape antitumor responses, resulting in immune escape of the tumor (33). The process of immune escape involves immunosuppressive factors, such as TGF- $\beta$ and IL-10, induction of dendritic cell (DC) apoptosis, changes in T cell subtypes and decreased interferon- $\gamma$ levels or its receptor expression. In detail, TGF- $\beta$ has a dual regulatory effect on tumors (34). During the initial stages of tumorigenesis, TGF- $\beta$ inhibits cell proliferation and initiates cell differentiation or apoptosis. However, during the progressive stage, this effect is lost, resulting in immune inhibition, stimulation of angiogenesis and induction of epithelia or mesenchymal transformation. This provides a favorable microenvironment for tumor cell proliferation, invasion and metastasis (31). IL-10, which plays a role in immunosuppression and promotes the immune escape of HCC cells, belongs to the Th2 type family of cytokines. For example, IL-10 can decrease the expression of the major histocompatibility complex II (MHC-II) and costimulatory molecules, such as CD80/86, thus weakening the functions of antigen-presenting cells (APCs) that kill tumor cells or indirectly kill tumor cells by activating T cells (30). As a result, HCC occurs.

\section{The role of PD1/PD-L1 in immune tolerance and immune escape of $\mathrm{HCC}$}

ICIs are a type of membrane binding molecule that play an important role in immune escape (35). In HCC, the common ICIs include PD-1/PD-L1, CTLA-4, lymphocyte activating gene 3 protein (LAG-3) and mucin domain molecule 3 (Tim-3) (26). PD-1, a member of the CD28 superfamily of proteins, is a costimulatory receptor expressed on immune cells, such as T, B and natural killer cells, but it is primarily expressed on the surface of activated $\mathrm{T}$ cells [CD8+ $\mathrm{T}$ cells, $\mathrm{T}$ regulatory cells (Tregs) or myeloid-derived suppressor cells] $(35,36)$. PD-L1 is a type I transmembrane protein composed of 290 amino acids, which can be upregulated on the surface of activated T cells, DCs and macrophages $(37,38)$. As an immunosuppressive receptor with a negative regulatory role, PD-1 always transmits a coinhibitory signal together with the T cell antigen receptor (TCR) after binding with PD-L1 and PD-L2 on the surface of activated T cells. Downstream signals gradually suppress the expression of genes required for $\mathrm{T}$ cell activation, which then induces tumor immune escape (39). In detail, the pathway composed of PD-1 and its ligands, PD-L1 and PD-L2, has a key role in maintaining peripheral immune tolerance (40). In turn, tumor cells also utilize this pathway to escape from T cell-induced immunity. PD-1 and PD-L1 or PD-L2, as two pairs of costimulatory signals, constitute the same pathway of PD-1-mediated signaling, which induces $\mathrm{T}$ cell activation and immune regulation by inhibiting cell proliferation $(41,42)$. When the PD-1/PD-L1 signaling pathway is activated, it reduces the damage of the immune response to surrounding tissues and helps prevent the occurrence of autoimmune diseases (40). Similarly, the activation of this pathway stimulates the binding of PD-L1 expressed by HCC cells to PD-1 on the surface of tumor infiltrating lymphocytes, inducing the apoptosis of specific cytotoxic T lymphocytes (CTL) that target the tumor. This decreases the activity of 


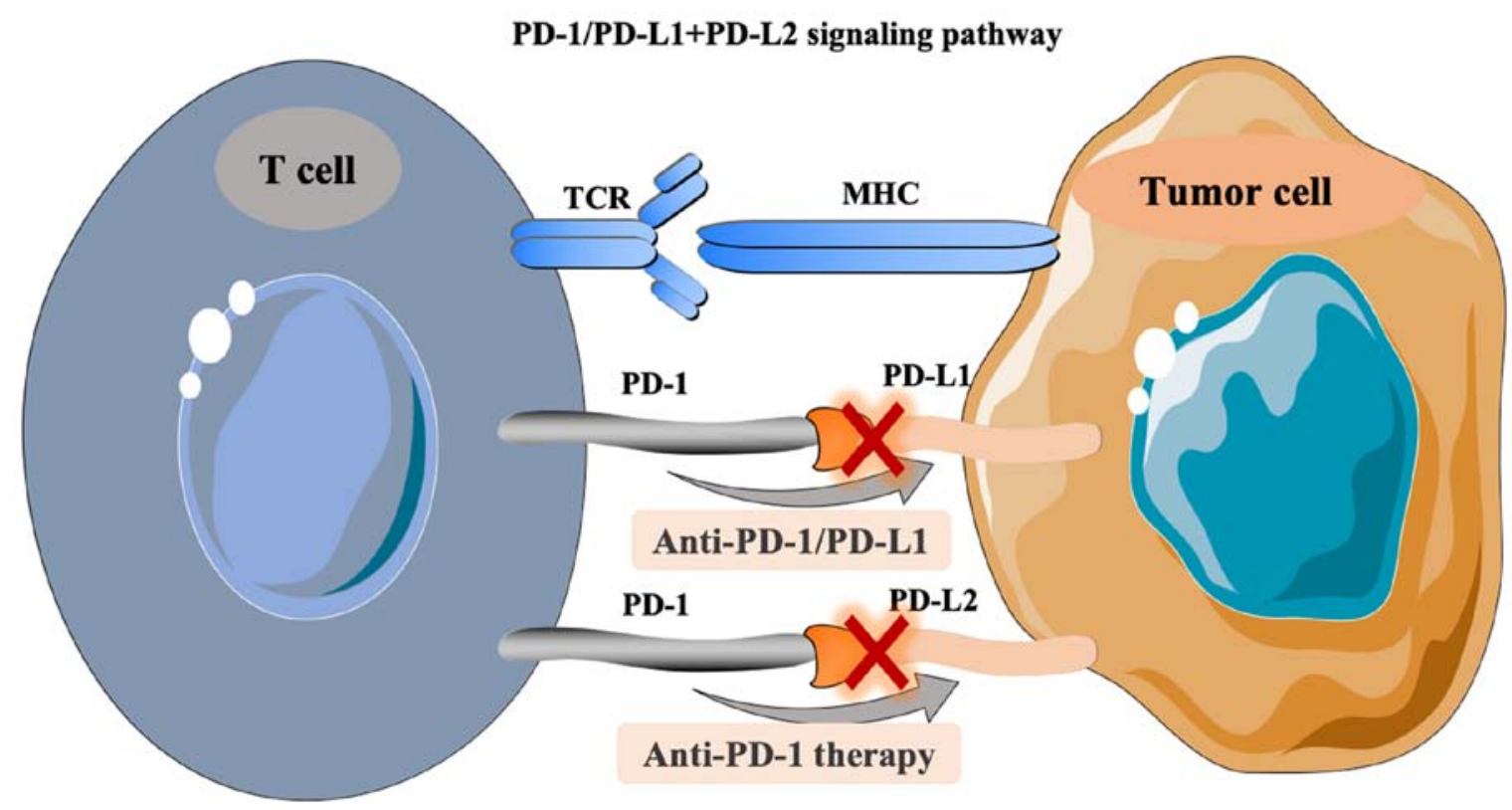

Figure 1. PD-1/PD-L1+PD-L2 signaling pathway. PD-1, programmed death receptor 1; PD-L1, programmed death ligand 1; TCR, T cell receptor; MHC, major histocompatibility complex.

$\mathrm{T}$ cells in the local microenvironment, and thus mediates immune escape (40). PD-L1 expression is upregulated in HCC (43). Some studies have demonstrated that this phenomenon induces the inherent expression of PD-L1 in tumor cells through the phosphatidylinositol 3 kinase- protein kinase B (PI3K-AKT) epidermal growth factor receptor and ALK tyrosine kinase receptor/STAT3 signaling pathways, thus regulating the expression of cell cycle checkpoint proteins and cell proliferation proteins $(33,34)$. Finally, the above process inhibits $\mathrm{T}$ cell proliferation $(17,44)$. Therefore, $\mathrm{PD}-1 / \mathrm{PD}-\mathrm{L} 1$ is a key interaction involved in the immune escape in liver cancer tumors $(26,36,39)$. In addition, the PD-1/PD-L1 antibody can suppress this immune escape ability, permitting cytotoxic $\mathrm{T}$ lymphocyte-mediated antitumor responses by blocking the binding of PD-L1 and PD-1 $(26,35,36)$. The treatment principle related to the PD-1/PD-L1 signaling pathway is shown in Fig. 1.

\section{Application of PD-1/PD-L1 inhibitors in HCC}

At present, the most commonly used PD-1 inhibitors are nivolumab, pembrolizumab, camrelizumab and sintilimab, while the common PD-L1 inhibitors include durvalumab and atezolizumab (37). Several guidelines have recommended immunotherapy of HCC. Among them, the National Comprehensive Cancer Network guidelines have recommended nivolumab and pembrolizumab as second-line treatment options for advanced HCC (45). The European Society of Oncology (ESMO) guidelines have recommended nivolumab as first-line and second-line options for HCC, and pembrolizumab has been recommended as a second-line option (6).

\section{Monotherapy studies}

Nivolumab. The approval of nivolumab in September 2017 was based on the CheckMate 040 trial (NCT01658878), which was a phase I/II single-arm, multi-center, multi-cohort clinical trial involving patients with advanced HCC (46). The patients enrolled onto the study included those who had or had not received sorafenib treatment and were $\mathrm{HBV}$ or $\mathrm{HCV}$ infected or not infected. In addition, the study had five cohorts: Cohorts 1 and 2 underwent the dose escalation phase $(0.1-10 \mathrm{mg} / \mathrm{kg}$ nivolumab was given once every 2 weeks) and dose expansion phase ( $3 \mathrm{mg} / \mathrm{kg}$ nivolumab was given once every 2 weeks until the disease progressed or toxic reactions could not be tolerated), respectively. The two cohorts included 262 patients with unresectable advanced HCC, including 182 patients who had previously received sorafenib treatment and 80 patients who had not, of whom 48 and 214 were in cohort 1 and 2, respectively. The results show that the objective response rate (ORR) was $16-19 \%$ and the median overall survival (mOS) time was 15.6 months. The ORR and mOS of the dose escalation group were $15 \%$ and 15 months, respectively, and those in the dose expansion group were $20 \%$ and 15.6 months, respectively. However, for other patients who had not received sorafenib as a first-line agent, the ORR and mOS were $20-23 \%$ and 28.6 months, respectively. It is noteworthy that in this trial, there were 85 patients from Asia; and the mOS of the total population in this trial was $14.9,14.8$ months for patients with HBV and HCV and 16.9 months for patients without these viruses. The study indicated that patients can benefit from nivolumab, regardless of hepatitis history. Moreover, the mOS of patients with stable disease (SD) and partial response (PR) were 17.5 and 27.4 months, respectively, further suggesting that those with SD may continue to benefit after immunotherapy (47). Furthermore, no viral recurrence was observed in the patients with $\mathrm{HBV} / \mathrm{HCV}$ infection.

Another global multicenter, randomized phase III clinical trial, CheckMate 459 (NCT02576509), was designed to observe the efficacy and safety of nivolumab as a first-line treatment for unresectable HCC. The study compared the efficacy of nivolumab with sorafenib to determine the safety and efficacy 
of nivolumab (48). Compared with sorafenib, the preliminary OS difference failed to reach the pre-set statistical significance threshold value [hazard ratio $(\mathrm{HR})=0.84 ; \mathrm{P}=0.0419$ ] and showed significant OS improvement $(\mathrm{HR}=0.85$; $95 \%$ confidence interval, 0.72-1.02; $\mathrm{P}=0.0752$ ). The 12 -month $\mathrm{OS}$ rate in the nivolumab group was $59.7 \%$, while that of the sorafenib group was $55.1 \%$. However, those patients in sorafenib group showed a notable trend towards OS prolongation.

The CheckMate 9DX trial (NCT03383458) focused on adjuvant immunotherapy after surgery (49). It aims to evaluate the efficacy of adjuvant nivolumab treatment compared with a placebo in patients with high risks of recurrence after HCC surgery, and it was estimated to be completed in 2025 .

Pembrolizumab. The FDA approved the use of pembrolizumab in November 2018 based on the results of the phase II single-arm, non-randomized trial, KEYNOTE-224 (NCT02702414) (50), which included 104 patients with HCC who had been previously treated with sorafenib. One patient achieved a complete response (CR), 17 patients achieved PR and 44 patients achieved SD. In addition, for the total population, the ORR was $17 \%$, and the disease control rate (DCR) was $62 \%$. Moreover, the median progression free survival (PFS) time was 4.9 months, while the OS time was 12.9 months.

In 2019, the American Society of Clinical Oncology (ASCO) meeting reported the results of the KEYNOTE-240 study (NCT02702401), which was a randomized, placebo-controlled phase III clinical study that evaluated the efficacy of pembrolizumab compared with the best supportive treatments, such as symptomatic palliation in patients with advanced HCC (51). In total, 413 patients were randomly assigned to the pembrolizumab group $(n=278)$ or the placebo group $(n=135)$ at a $2: 1$ ratio. The results showed that after a median follow-up of 13.8 months, $10.1 \%$ of the experimental group and $3.0 \%$ of the placebo group were still receiving therapy. Compared with the placebo group, the OS time in the test group was prolonged by 3 months (13.9 months vs. 10.6 months; $\mathrm{HR}=0.78$; unilateral $\mathrm{P}=0.0238)$, but this figure did not reach the established statistical difference (25). However, in the OS and PFS subgroup analyses, the majority of subgroups observed an obvious prolonging trend for pembrolizumab, thus suggesting clinical benefits. Furthermore, the ORRs of the pembrolizumab group and placebo group were $18.3 \%$ (95\% CI, 14.0-23.4) and 4.4\% (95\% CI, 1.6-9.4), respectively. Regarding safety, common adverse events included an increase in the levels of transaminase and bilirubin, fatigue, pruritus, loss of appetite and diarrhea. The incidence of grade $3 / 4$ toxicities was relatively low. Another phase III (NCT03062358), randomized trial is currently being conducted.

Camrelizumab. At the ESMO meeting in October 2018, a multicenter, open, randomized, parallel-controlled, phase II clinical trial(NCT02989922) in China that enrolled 220 patients (data for 217 patients can be accessed) was reported. In detail, patients were randomly assigned to two subgroups at the ratio of $1: 1$ and received intravenous camrelizumab $(3 \mathrm{mg} / \mathrm{kg})$ every 2 weeks (q2w, n=111) and every 3 weeks (q3w, n=109), respectively. The overall ORR was $13.8 \%$ (30/217), and the 6-month OS rate was $74.7 \%$ (52).

In September 2019, the data of the NCT02989922 study were updated at the meeting of the Chinese Society of Clinical
Oncology (CSCO) (52). The ORR of all subjects was $14.7 \%$, while the 6-month OS rate was $74.4 \%$. Meanwhile, the most common adverse event was reactive capillary epithelial proliferation (RCREP), the incidence of which was $\sim 66.8 \%$ (52).

In April 2020, Qin et al updated the latest results of NCT02989922 in China (53). By November 2018, the median follow-up time was 12.5 months. The results showed that both the $\mathrm{q} 2 \mathrm{w}$ and $\mathrm{q} 3 \mathrm{w}$ subgroups showed a higher ORR of 11.9 and $17.6 \%$, respectively, and the ORR was $14.7 \%$ among all patients. In addition, the 6-month and 12-month OS rates were 74.4 and $55.9 \%$, respectively. As for safety, the most common treatment-associated adverse events (AEs) was still RCREP with an incidence of $67 \%$. The incidence of grade 3-4 AEs was $22 \%$. Both the ORR and the incidence of AEs were similar to that of other immune drugs, which is why camrelizumab was approved as the first second-line ICI agent for the treatment of HCC in China (53).

\section{Combination therapy studies}

Camrelizumab plus apatinib. A phase I clinical trial (NCT02942329) investigating the combination of camrelizumab and apatinib [a TKI selectively acting on vascular endothelial growth factor receptor (VEGFR)2] was reported at the ASCO meeting in June 2018 (54). The results showed that the ORR of 14 patients was $\sim 50 \%$, and the DCR was $85.7 \%$, which indicated that the binding of PD-1 with antiangiogenic drugs may have a synergistic effect in advanced HCC. The primary and most serious complication was hypertension.

Camrelizumab plus chemotherapy. In September 2018, the CSCO conference reported the results of a study performed by Qin et al. This was a phase II clinical trial investigating first-line treatment with camrelizumab combined with the FOLFOX regimen (fluorouracil+calcium folinate+oxaliplatin) in HCC. In 22 patients, the ORR was $27.3 \%$, and the DCR was $72.7 \%$. Furthermore, a phase III clinical study (NCT03605706), required to further confirm the efficacy and safety, is recruiting participants and is estimated to complete in 2021. However, the results have not yet been reported. The primary outcome is OS.

Moreover, Qin et al registered a multicenter phase II trial to assess the efficacy and safety of camrelizumab combined with FOLFOX4 as a first-line treatment in advanced HCC (55). As a result, the ORR and DCR of 34 patients were 26.5 and $79.4 \%$, respectively. Although the mOS level was not achieved, the safety of combinational therapy was controllable, and the local control profiles of tumor were good mainly reflected in DCR.

Atezolizumab plus bevacizumab. Atezolizumab targets PD-L1 (56). A phase IB clinical study of atezolizumab combined with bevacizumab for the treatment of advanced HCC, the GO30140 study (NCT02715531) (56), was reported at the ASCO conference in 2018. In 23 assessable patients, the ORR was $65 \%$ and the DCR was $95 \%$.

In November 2019, the IMbrave 150 study, a global multi-center and open-label phase III trial that enrolled 501 patients with unresectable HCC who did not receive systemic therapies, was reported at the ESMO Asian conference (Abstract: LBA3) (57). The patients were randomly 


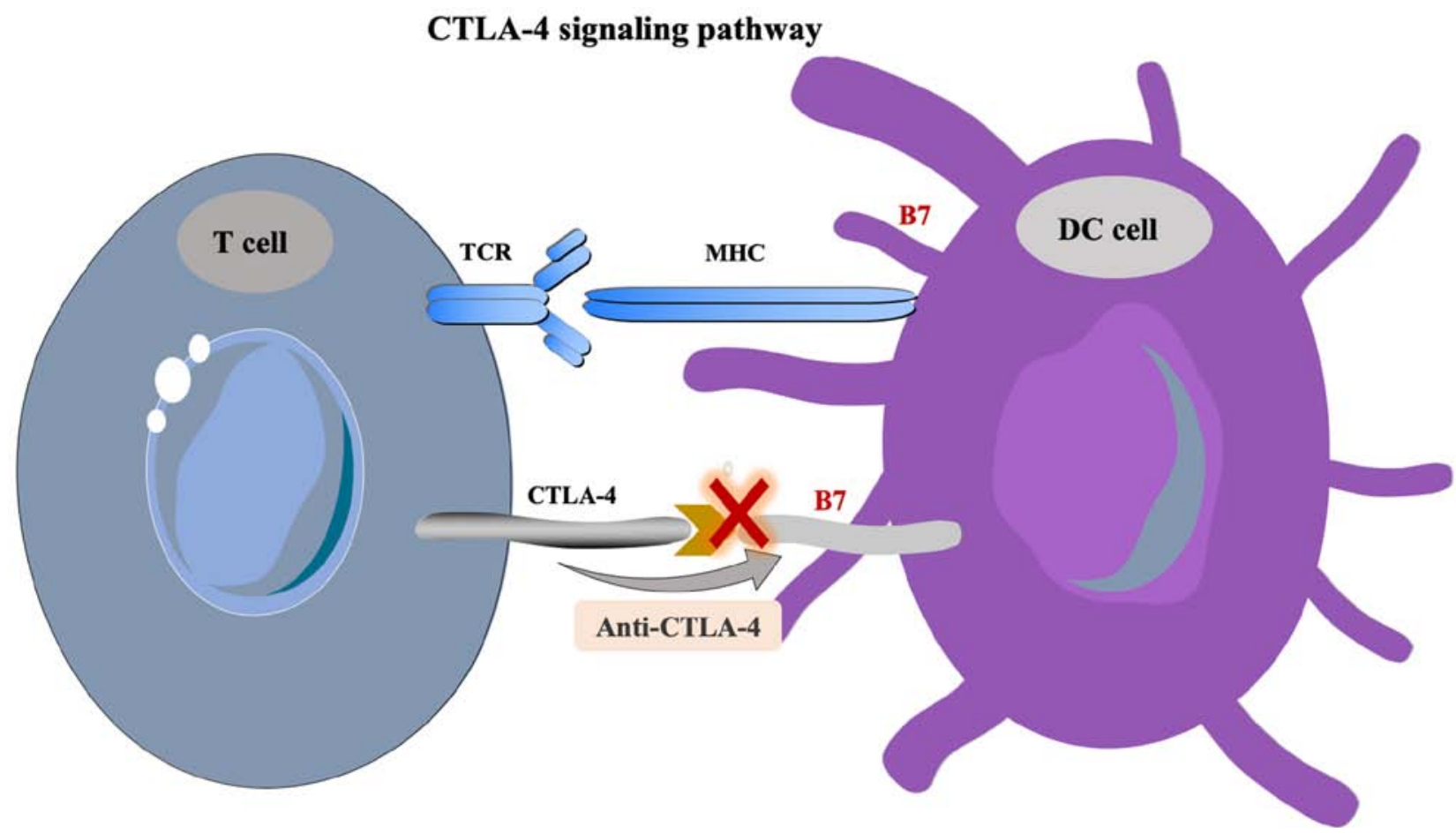

Figure 2. CTLA-4 signaling pathway. CTLA-4, cytotoxic T lymphocyte-associated protein 4; TCR, T cell receptor; DC, dendritic cell; MHC, major histocompatibility complex.

administered atezolizumab combined with bevacizumab or sorafenib at a 2:1 ratio until unacceptable toxicity or no further clinical benefits were observed. The latest results showed that the median PFS time in the atezolizumab group was 6.8 months (range, 5.7-8.3 months), while that of the sorafenib group was 4.3 months (range, 4.0-5.6 months).

At the Liver Cancer Summit 2020, which is organized by the European Association for the Study of the Liver, Qin et al presented the Chinese subgroup results of the IMbrave trial, which confirmed the previous global results (58). Among 194 Chinese patients who had poorer prognostic factors compared with the global data, 133 patients were randomly assigned to the combination group (atezolizumab plus bevacizumab) and 61 to the sorafenib group. The median follow-up time of the combined group and the sorafenib group was 7.2 and 5.6 months, respectively. The mPFS was 5.7 vs. 3.2 months, and the ORR was 25 vs. $7 \%$, which was consistent with the global results. Moreover, the incidence of toxicity was relatively low.

Pembrolizumab plus lenvatinib. In the REFLECT study, which aimed to compare the efficacy of lenvatinib and sorafenib in unresectable patients with advanced HCC, lenvatinib was shown not to be inferior to sorafenib; therefore, lenvatinib was approved as a first-line agent for advanced HCC (59). The 2018 ASCO meeting reported a phase IB trial that enrolled 30 patients to evaluate the safety and efficacy of pembrolizumab and lenvatinib in advanced HCC (60). Among the 26 patients who could be included for evaluation, results showed that one patient achieved CR, 10 achieved PR and 15 achieved SD. Moreover, the ORR was 42.3\%, and the PFS time reached 9.69 months. In 2019, the American Association for Cancer Research updated the data. Compared with the previous results in the 2018 ASCO meeting (60), the number of patients achieving CR was three, while 15 patients achieved PR according to the modified Response Evaluation Criteria in Solid Tumors (RECIST) standards (61). Indeed, these data showed encouraging trends, in particular, the increase of patients who reached PR (from 10 to 15). Therefore, a global phase III randomized, controlled clinical study investigating first-line treatment for advanced HCC using pembrolizumab and lenvatinib has been launched, with pending results.

In 2019, Llovet et al updated the results concerning combination therapy of pembrolizumab and lenvatinib in advanced HCC (62). The results showed that tolerance of the combination of agents was acceptable and only $5 \%$ of patients had to terminate treatment because of grade 3-4 AEs. The ORR and DCR was 31 and $49 \%$, respectively, and the 1-year survival rate was $40 \%$.

Nivolumab plus lenvatinib. At the ASCO-GI 2020 meeting, Kudo et al (63) reviewed the data of a phase IB trial that focused on the efficacy of nivolumab combined with lenvatinib in advanced HCC. The study enrolled 30 patients who were randomly divided into two groups: One group $(n=6)$ had multidrug resistance and the other group $(n=24)$ had no previous treatments. The first part of this study explored the dose tolerance of combinational agents via DLT evaluation. The results showed that no DLT was observed in six patients. Among the 30 patients, the ORR was $76.7 \%$ and the DCR was $96.7 \%$. Moreover, $\sim 10 \%$ of patients achieved a complete response (CR). However, the second part of this study enrolled 24 patients with no prior systematic therapy for unresectable HCC who all received nivolumab (200 $\mathrm{mg}$ ) plus lenvatinib (12 $\mathrm{mg}$ or $8 \mathrm{mg}$, according to the weight of patient), and for these patients the ORR was $79.2 \%$. 


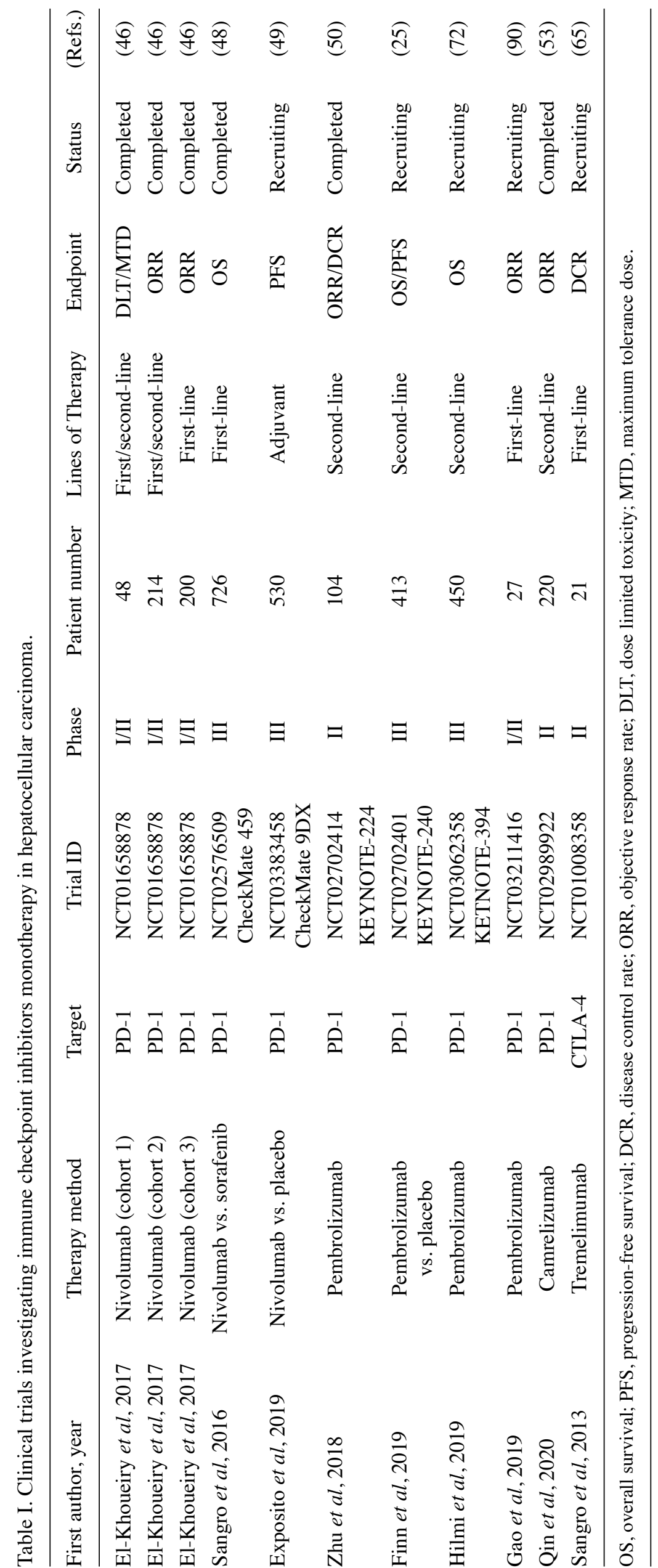


Sintilimab plus IBI305. The ORIENT 32 study (NCT03794440) is an open-label, multi-centre trial in China in which patients are randomized to receive a combination of sintilimab and recombinant anti-VEGF humanized monoclonal antibody (IBI305) vs. sorafenib (23). However, the results have not yet been published.

\section{The role of CTLA-4 in immune tolerance and immune escape of $\mathrm{HCC}$}

CTLA-4 is a type of transmembrane receptor on T cells that is primarily expressed on the surface of activated $\mathrm{T}$ cells (42). The activation of $\mathrm{T}$ lymphocytes requires the activation of two signaling pathways, including the binding of the T cell receptor (TCR) and MHC-peptide complex, which is presented by APCs and the B7 molecule to the costimulatory molecule $\mathrm{CD} 28$ on the surface of $\mathrm{T}$ cells (17). CTLA-4, which always shares the B7 molecular ligand of the APC with CD28, is highly expressed in Tregs and activated $\mathrm{T}$ lymphocytes, inducing the unresponsiveness of $\mathrm{T}$ cells to negatively regulate the immune response (19). The overexpression of CTLA-4 in HCC leads to uncontrolled growth of the tumor (64). CTLA-4 inhibitors can block the activation of the CTLA-4 pathway, enhance the activation and proliferation of $\mathrm{T}$ cells and then lower Treg-mediated immunosuppression (17). The treatment principle concerning the CTLA-4 signaling pathway is shown in Fig. 2.

\section{Applications of CTLA-4 inhibitors in HCC}

Stimulated by the TCR, CTLA- 4 can be jointly employed with $\mathrm{B} 7$ molecules to produce inhibition signals, thus inhibiting the activation of T cells and then decreasing their ability to recognize tumor antigens. This promotes the occurrence and development of tumors (64). CTLA-4 inhibitors restore the immune activity of $\mathrm{T}$ cells by blocking the binding of CTLA-4 to B7 molecules $(17,21,64)$, and inhibitors include tremelimumab and ipilimumab.

Tremelimumab is a type of human immunoglobulin G 2 monoclonal antibody that blocks the signaling pathway involving CTLA-4 (65). Sangro et al reported a small-sample phase II clinical trial of tremelimumab in 2013 (NCT01008358), in which 21 patients with $\mathrm{HCV}$-associated HCC who received tremelimumab $(15 \mathrm{mg} / \mathrm{kg}$; every 90 days; maximum dose, 4 times) were recruited (65). There were 17 patients who were incorporated into the evaluation. The study demonstrated that tremelimumab has antitumor effects and certain antiviral activity. In detail, the partial response (PR) rate was $17.6 \%(3 / 17)$, and the DCR was $76.4 \%$ (13/17). Besides, the mOS was 8.2 months, and the time to progression was 6.48 months. Moreover, no serious adverse events were observed.

PD-1/PD-L1 inhibitors combined with CTLA-4 inhibitors in $H C C$

Combination of nivolumab and ipilimumab. Based on the CheckMate 040 study (NCT01658878) (66), cohort 4 was used to explore the safety and efficacy of the combination of nivolumab and ipilimumab in patients with HCC 
previously treated with sorafenib (67). This trial included 148 patients, $\sim 88 \%$ of whom had vascular invasion or extrahepatic metastasis and $91 \%$ of whom were diagnosed as having BCLC stage $\mathrm{C}$ disease. According to the results, the ORR was $31 \%$ and the DCR was $49 \%$. The OS of patients who received maintenance therapy (nivolumab $1 \mathrm{mg} / \mathrm{kg}$, ipilimumab $3 \mathrm{mg} / \mathrm{kg}$ and sequential nivolumab therapy $240 \mathrm{mg}$ ) was 22.8 months. The safety profile analysis suggested that this combination of double immunosuppressive agents was well tolerated as there were relatively few grade 3/4 treatment-associated AEs, and novel AEs were observed in the experimental group after the increase in ipilimumab dose. According to the encouraging data of CheckMate 040 study, in March 2020, the combination of nivolumab and ipilimumab was approved for patients with HCC who previously treated with sorafenib by FDA (68).

Durvalumab monotherapy or combination therapy with tremelimumab. In advance, the HIMALAYA study (NCT03298451) (69), an open-label, multicenter and randomized phase III study investigating durvalumab monotherapy or combined therapy with tremelimumab vs. sorafenib in advanced HCC, had planned to enroll 1,350 patients. In 2017 , Kelley et al (70) reported the early data of 40 patients in a phase I trial investigating the combination of durvalumab with tremelimumab in advanced HCC. Additionally, other phase I/II trials (NCT03222076 and NCT03203304) have been designed to evaluate the efficacy of a combination of immune checkpoint blockers in advanced HCC (71-73).

\section{Combination immunotherapy with locoregional therapy in $\mathrm{HCC}$}

Similar to targeted drugs, local treatments can mechanically reinforce the efficacy of ICIs by stimulating the release of tumor-associated antigens from the tumor cells (29). In addition, the combination of radiotherapy and chemotherapeutic agents is expected to increase neoantigen release through DNA interference. This may lead to the improved efficacy of ICIs, induce immunogenic cell death and enhance the immune response by decreasing the number of immunosuppressive cells, such as Tregs and myelogenous suppressor cells $(29,74)$.

Combination of TACE via drug-eluting bead (DEB-)TACE and nivolumab. In 2018, the ASCO meeting reported a multicenter, phase I trial of nivolumab with DEB-TACE in unresectable HCC that aimed to evaluate the safety and tolerability of combined therapies. The IMMUTACE study (NCT03572582) was a phase II trial in Germany focusing on DEB-TACE and nivolumab (75).

Combination of TACE or RFA and pembrolizumab. NCT03397654, a phase I/II study, was designed to assess the safety and efficacy of the combination therapy of TACE (using doxorubicin, $60 \mathrm{mg}$ ) plus pembrolizumab (200 mg, starting 30 or 45 days after TACE) that was repeatedly at three-week intervals (73). The IMMULAB study investigating the combination of local RFA plus pembrolizumab is currently ongoing.
Combination of RFA and tremelimumab. In 2017, Duffy et al (65) reported a study that totally enrolled 32 patients with HCC who received tremelimumab (two-dose level, $3.5 \mathrm{mg} / \mathrm{kg}$ and $10 \mathrm{mg} / \mathrm{kg}$ ) every 4 weeks, and then followed by RFA. In total, $26 \%$ of patients achieved PR. In addition, the 6-month and the 12-month PFS rates were 57.1 and $33.1 \%$, respectively, whilst the mOS was 12.3 months. Tables I and II showed the current clinical trials investigating ICIs in HCC.

\section{Current concerns}

At present, the concerns pertaining to HCC therapy are as following.

Response of potential immunotherapy biomarkers in HCC. According to the CheckMate 040 trial, it was reported that patients with $\mathrm{HCC}$ can benefit from nivolumab regardless of a history of hepatitis or PD-L1 expression in tumor cells. There is no significant difference in the efficacy between Asian patients and global patients, such as the OS and PFS trends $(46,47)$. Therefore, in contrast to the previous hypothesis that PD-L1 was a predictive biomarker (76), it is not necessary to detect PD-L1 expression in tumor tissue when choosing nivolumab as a second-line treatment in HCC (46). Previous studies (REACH and REACH-2 study) have indicated that the baseline level of AFP is associated with the efficacy of ramucirumab (CYRAMZA trial) (77,78). However, the CheckMate 040 and the KEYNOTE-224 studies reported that the efficacy of nivolumab and pembrolizumab cannot be predicted, suggesting that there are no effective biomarkers to predict the treatment response of PD-1 inhibitors in HCC $(50,66)$. At present, the primary immunotherapy biomarker factors undergoing further research are microsatellite stability, tumor mutation load, immune cell status (CD4+ or CD8+ T cells) (29,79), immunosuppressive receptors (TIM-3 and LAG-3) $(29,80)$ and immunosuppressive enzymes (Indoleamine 2,3-dioxygenase, adenosine pathway) (79). Therefore, specific biomarkers that can be used to predict clinical efficacy of PD-1 inhibitors are needed.

The loss of mismatch repair (MMR) genes can typically cause the accumulation of mismatch in the process of DNA replication, leading to the occurrence of microsatellite instability (MSI) (81). MSI is common in gastrointestinal tumors such as gastric adenocarcinoma (in 15-20\% of cases) and colorectal adenocarcinoma (in $12-15 \%$ of cases), especially in hereditary nonpolyposis hereditary colorectal cancer or Lynch syndrome, which normally characterized by deficient MMR and/or microsatellite instability-high (MSI-H) (82). As previous studies have reported, patients with MSI-H treated with immunotherapies always show a higher response rate and improved efficacy compared with those patients without MSI-H. Therefore, several immunotherapeutic agents, such as pembrolizumab, have been approved to treat solid tumors including $\mathrm{HCC}$ (83). However, the incidence of MSI-H in HCC is $2 \%$ (84-86). Also, the response rate of patients with HCC to pembrolizumab is quite low; $2-2.4 \%(84,86)$. Moreover, the association between MSI and immune checkpoints are unclear and thus need further investigating.

Evaluation standard of immunotherapy response. Unlike traditional chemotherapy or radiotherapy, considering the 
delay of therapeutic benefits and durable long-lasting effects of immunotherapy due to the initial recruitment of activated $\mathrm{T}$ cells to the tumor site before the initiation of antitumor activity, the assessment standard can be modified and optimized through short-term and long-term efficacy evaluation based on the RECIST guidelines. This enables investigators to accurately track and grasp the change of tumor during therapy $(39,87)$. In addition, prior studies investigating the application of ICIs in HCC mainly addressed their use as second-line therapy $(50,53,72)$. Hence, the use of PD-1 agents as first-line treatment should be addressed in future clinical trials.

\section{Conclusions}

In the past 10 years, sorafenib has been the only targeted drug approved by the FDA as a first-line treatment agent for advanced HCC (7). The overall prognosis of HCC remains quite poor (15). Despite the gradual emergence of TKIs, including regorafenib and lenvatinib (12-14), or local interventional therapies (9-11), substantial therapeutic advances in HCC are still lacking. Therefore, the discovery of immunotherapy, especially ICIs, provides new avenues for the comprehensive and systemic treatment of advanced HCC. Mechanistically, TKIs affect antigen presentation and the microenvironment, thereby enhancing or dampening the immune response by stimulating the release of tumor-associated antigens (7). Similarly, anti-angiogenesis drugs, such as bevacizumab, can also inhibit tumor growth by reducing the blood supply of tumor (12-14). Thus, TKIs or anti-angiogenesis agents can be synergistic with immunotherapeutic drugs. In addition, there are the potential benefits of the synergistic effects of TACE or RFA combined with immunotherapy (9-11). Therefore, combination treatments, not just limited to immunotherapy agents but also TKIs, anti-angiogenesis drugs and locoregional therapies, appears to be a novel and promising strategy.

Several of the latest published datasets presented in the ASCO meeting, although discouraging, indicated that the inclusion of ICIs in combination therapies rendered them relatively more effective, especially in terms of OS and PFS time $(25,55,66)$. For example, the negative results in KEYNOTE-240 may be due to the following reasons. First of all, there was an inappropriate study design related to this trial. For example, $\sim 47.4 \%$ of the placebo group subsequently received antitumor treatments, and $\sim 10.4 \%$ of them received PD-1/PD-L1 inhibitor treatments, which may have affected the final results (the P-value did not reach statistical significance). Hence, the effect of immunotherapy on placebo group cannot be ignored. In addition, the survival data for the placebo group were superior to those in other trials owing to the strict enrollment selection; that is, this study excluded numerous patients with macrovascular invasion that was regarded as one risk factor of HCC prognosis. Furthermore, the OS and the PFS were both set as endpoints resulting in higher requirements for achieving significant results. Besides, this study did not recruit Chinese patients who were the population with a high incidence of HCC; however, another study, KEYNOTE-394, is recruiting Chinese patients. Similar to KEYNOTE-240, CheckMate 459 also presented the negative results, mainly in the OS and the PFS which failed to reach the statistical difference $(\mathrm{HR}=0.85 ; \mathrm{P}=0.0752)$. However, the nivolumab group showed notably prolonged OS time compared with the sorafenib group (16.4 vs. 14.7 months, $\mathrm{P}=0.0752)$. Also, this study suggested that the response to nivolumab was associated with PD-L1 expression. In particular, those with PD-L1 $\geq 1 \%$ had higher response rate compared with those with PD-L1 $<1 \%$. Therefore, PD-L1 may be a predictive biomarker for the response to nivolumab treatment.

Further phase III trials should be conducted in the future to investigate the efficacy of combination therapies. Additional research is also needed to determine biomarkers to predict clinical efficacy. Similarly, the concern of selecting suitable patients should be addressed, such as the PD-L1 status. It is speculated that patients with PD-L1 (+) may be benefit from immunotherapies. Owing to the delayed effects of immunotherapies, the evaluation standards should take into account as well.

Additionally, HCC typically accompanies chronic HBV or HCV infection, especially in Asian countries, such as China (88). In 2019, Fisicaro et al reported that in patients with chronic hepatitis B infection, numbers of specific T cells are low and these cells are easily exhausted (89). Thus, these T cells, such as CD8+ T cells, always show a suppressive effect, which is due to environmental changes triggered by inflammation and dysregulation of immune receptor expression, including upregulation of multiple co-inhibitory receptors (89). Controlling the infection via anti-HBV/anti-HCV treatment would spontaneously induce an extensive and powerful response of antigen-specific T cells. Also, in patients HCC with $\mathrm{HBV}$ or $\mathrm{HCV}$ infection, the PD-1/PD-L1 signaling pathway can cause similar deactivation of specific CD8+ cells (89). Therefore, specifically blocking the PD-1/PD-L1 or B7-CTLA-4 signaling pathway via ICIs theoretically can restore the activity of $\mathrm{T}$ cells, therefore this may be helpful in controlling the virus and tumor progression in these patients. Therefore, patients with $\mathrm{HBC} / \mathrm{HVC}$-associated HCC may have an improved response to immunotherapies compared to those without hepatitis virus infection. However, the application of ICIs in hepatitis virus infection is quite limited $(5,89)$. This is possibly associated with the poor selectivity of ICIs and the liver damage mediated by the suppression of normal liver tissue function via co-inhibitory pathways; therefore, one potential solution is to silence the inhibitory paths and to restore the activity and function of virus-specific $\mathrm{T}$ cells such as CD8+ T cells. In the future, more research is needed to investigate virus-associated and non-viral HCC.

Overall, using ICIs as the first-line treatment or combined with other therpies such as local regional methods and targeted agents in future trial is a noteworthy point. Also, seeking potential biomarkers contributes to predicting the therapeutic effect and filtering suitable participants for immunotherapies. In conclusion, immunotherapy in $\mathrm{HCC}$ is indeed promising but challenging.

\section{Acknowledgements}

The authors would like to thank Dr FangYun Yang (Department of Abdominal Oncology, Cancer Center, West China Hospital, West China Medical School, Sichuan University) and Dr Biao Yang (Departments of Gastroenterology, West China Hospital, 
West China Medical School, Sichuan University) for their support.

\section{Funding}

This study was sponsored by the Department of Science \& Technology of Sichuan Province of China (2017SZ0014) to LZY.

\section{Availability of data and materials}

Not applicable.

\section{Authors' contributions}

ZYL contributed to the conception and idea of this article. $\mathrm{ZZ}$ and BY contributed to the literature search. $\mathrm{ZZ}$ wrote the manuscript.

\section{Ethics approval and consent to participate}

Not applicable.

\section{Patient consent for publication}

Not applicable.

\section{Competing interests}

The authors declare that they have no competing interests.

\section{References}

1. McGlynn KA, Petrick JL and London WT: Global Epidemiology of Hepatocellular Carcinoma: An emphasis on demographic and regional variability. Clin Liver Dis 19: 223-238, 2015.

2. Miller KD, Nogueira L, Mariotto AB, Rowland JH, Yabroff KR, Alfano CM, Jemal A, Kramer JL and Siegel RL: Cancer treatmen and survivorship statistics, 2019. CA Cancer J Clin 69: 363-385, 2019.

3. Zeng H, Chen W, Zheng R, Zhang S, Ji JS, Zou X, Xia C, Sun K, Yang Z, Li H, et al: Changing cancer survival in China during 2003-15: A pooled analysis of 17 population-based cancer registries. Lancet Glob Health 6: e555-e567, 2018.

4. Kanwal F, Kramer J, Asch SM, Chayanupatkul M, Cao Y and El-Serag HB: Risk of hepatocellular cancer in HCV patients treated with direct-acting antiviral agents. Gastroenterology 153: 996-1005. e1, 2017.

5. Tan AT, Yang N, Lee Krishnamoorthy T, Oei V, Chua A, Zhao X, Tan HS, Chia A, Le Bert N, Low D, et al: Use of expression profiles of HBV-DNA integrated into genomes of hepatocellular carcinoma cells to select $\mathrm{T}$ cells for immunotherapy. Gastroenterology 156: 1862-1876.e9, 2019.

6. Vogel A, Cervantes A, Chau I, Daniele B, Llovet JM, Meyer T, Nault JC, Neumann U, Ricke J, Sangro B, et al: Hepatocellular carcinoma: ESMO clinical practice guidelines for diagnosis, treatment and follow-up. Ann Oncol 30: 871-873, 2019.

7. Benson AB III, D'Angelica MI, Abbott DE, Abrams TA, Alberts SR Saenz DA, Are C, Brown DB, Chang DT, Covey AM, et al: NCCN guidelines insights: Hepatobiliary cancers, version 1.2017. J Natl Compr Canc Netw 15: 563-573, 2017.

8. Bruix J, Reig M and Sherman M: Evidence-based diagnosis, staging, and treatment of patients with hepatocellular carcinoma. Gastroenterology 150: 835-853, 2016.

9. Kudo M, Ueshima K, Ikeda M, Torimura T, Tanabe N, Aikata H, Izumi N, Yamasaki T, Nojiri S, Hino K, et al: Randomised, multicentre prospective trial of transarterial chemoembolisation (TACE) plus sorafenib as compared with TACE alone in patients with hepatocellular carcinoma: TACTICS trial. Gut 69: 1492-1501, 2020 .
10. Cui J, Wang N, Zhao $\mathrm{H}$, Jin $\mathrm{H}$, Wang $\mathrm{G}$, Niu $\mathrm{C}$, Terunuma $\mathrm{H}$ $\mathrm{He} \mathrm{H}$ and $\mathrm{Li}$ W: Combination of radiofrequency ablation and sequential cellular immunotherapy improves progression-free survival for patients with hepatocellular carcinoma. Int $\mathrm{J}$ Cancer 134: 342-351, 2014.

11. Lencioni R, Llovet JM, Han G, Tak WY, Yang J, Guglielmi A, Paik SW, Reig M, Kim DY, Chau GY, et al: Sorafenib or placebo plus TACE with doxorubicin-eluting beads for intermediate stage HCC: The SPACE trial. J Hepatol 64: 1090-1098, 2016.

12. Abou-Alfa GK, Meyer T, Cheng AL, El-Khoueiry AB Rimassa L, Ryoo BY, Cicin I, Merle P, Chen Y, Park JW, et al: Cabozantinib in patients with advanced and progressing hepatocellular carcinoma. N Engl J Med 379: 54-63, 2018.

13. Bruix J, Qin S, Merle P, Granito A, Huang YH, Bodoky G, Pracht M, Yokosuka O, Rosmorduc O, Breder V, et al: Regorafenib for patients with hepatocellular carcinoma who progressed on sorafenib treatment (RESORCE): A randomised, double-blind, placebo-controlled, phase 3 trial. Lancet 389: 56-66, 2017.

14. Llovet JM, Ricci S, Mazzaferro V, Hilgard P, Gane E, Blanc JF, de Oliveira AC, Santoro A, Raoul JL, Forner A, et al: Sorafenib in advanced hepatocellular carcinoma. N Engl J Med 359: 378-390, 2008.

15. Greten TF, Lai CW, Li G and Staveley-O'Carroll KF: Targeted and immune-based therapies for hepatocellular carcinoma. Gastroenterology 156: 510-524, 2019.

16. Maur M, Tomasello C, Frassoldati A, Dieci MV, Barbieri E and Conte P: Posterior reversible encephalopathy syndrome during ipilimumab therapy for malignant melanoma. J Clin Oncol 30: e76-e78, 2012.

17. Buchbinder EI and Desai A: CTLA-4 and PD-1 Pathways: Similarities, differences, and implications of their inhibition. Am J Clin Oncol 39: 98-106, 2016.

18. Sprinzl MF and Galle PR: Current progress in immunotherapy of hepatocellular carcinoma. J Hepatol 66: 482-484, 2017.

19. Prieto J, Melero I and Sangro B: Immunological landscape and immunotherapy of hepatocellular carcinoma. Nat Rev Gastroenterol Hepatol 12: 681-700, 2015.

20. Wang L and Wang FS: Clinical immunology and immunotherapy for hepatocellular carcinoma: Current progress and challenges. Hepatol Int 13: 521-533, 2019.

21. Kudo M: Immuno-oncology in hepatocellular carcinoma: 2017 Update. Oncology 93 (Suppl 1): S147-S159, 2017.

22. Waidmann O: Recent developments with immunotherapy for hepatocellular carcinoma. Expert Opin Biol Ther 18: 905-910, 2018.

23. Johnston MP and Khakoo SI: Immunotherapy for hepatocellular carcinoma: Current and future. World J Gastroenterol 25: 2977-2989, 2019

24. Tian M, Shi Y, Liu W and Fan J: Immunotherapy of hepatocellular carcinoma: Strategies for combinatorial intervention. Sci China Life Sci 62: 1138-1143, 2019.

25. Finn RS, Ryoo BY, Merle P, Kudo M, Bouattour M, Lim HY, Breder VV, Edeline J, Chao Y, Ogasawara S, et al: Results of KEYNOTE-240: Phase 3 study of pembrolizumab (Pembro) vs best supportive care (BSC) for second line therapy in advanced hepatocellular carcinoma (HCC). J Clin Oncol 37 (15 Suppl): S4004, 2019.

26. Eggert T and Greten TF: Tumor regulation of the tissue environment in the liver. Pharmacol Ther 173: 47-57, 2017.

27. Nishida $\mathrm{N}$ and Kudo $\mathrm{M}$ : Immunological microenvironment of hepatocellular carcinoma and its clinical implication. Oncology 92 (Suppl 1): S40-S49, 2017.

28. Tiegs G and Lohse AW: Immune tolerance: What is unique about the liver. J Autoimmun 34: 1-6, 2010.

29. Huz JI, Melis M and Sarpel U: Spontaneous regression of hepatocellular carcinoma is most often associated with tumour hypoxia or a systemic inflammatory response. HPB (Oxford) 14: 500-505, 2012.

30. Crispe IN: Liver antigen-presenting cells. J Hepatol 54: 357-365, 2011.

31. Jiang Y, Han QJ and Zhang J: Hepatocellular carcinoma: Mechanisms of progression and immunotherapy. World $\mathrm{J}$ Gastroenterol 25: 3151-3167, 2019.

32. Josefowicz SZ, Lu LF and Rudensky AY: Regulatory T cells: Mechanisms of differentiation and function. Annu Rev Immunol 30: 531-564, 2012.

33. Gong J, Chehrazi-Raffle A, Reddi S and Salgia R: Development of PD-1 and PD-L1 inhibitors as a form of cancer immunotherapy: A comprehensive review of registration trials and future considerations. J Immunother Cancer 6: 8, 2018. 
34. Zou W, Wolchok JD and Chen L: PD-L1 (B7-H1) and PD-1 pathway blockade for cancer therapy: Mechanisms, response biomarkers, and combinations. Sci Transl Med 8: 328rv4, 2016.

35. Calderaro J, Rousseau B, Amaddeo G, Mercey M, Charpy C, Costentin C, Luciani A, Zafrani ES, Laurent A, Azoulay D, et al: Programmed death ligand 1 expression in hepatocellular carcinoma: Relationship with clinical and pathological features. Hepatology 64: 2038-2046, 2016.

36. Wei F, Zhong S, Ma Z, Kong H, Medvec A, Ahmed R, Freeman GJ, Krogsgaard M and Riley JL: Strength of PD-1 signaling differentially affects T-cell effector functions. Proc Natl Acad Sci USA 110: E2480-E2489, 2013

37. El Dika I, Khalil DN and Abou-Alfa GK: Immune checkpoint inhibitors for hepatocellular carcinoma. Cancer 125: 3312-3319, 2019.

38. Noonan A and Pawlik TM: Hepatocellular carcinoma: An update on investigational drugs in phase I and II clinical trials. Expert Opin Investig Drugs 28: 941-949, 2019.

39. Mizukoshi E and Kaneko S: Immune cell therapy for hepatocellular carcinoma. J Hematol Oncol 12: 52, 2019.

40. Liang SC, Latchman YE, Buhlmann JE, Tomczak MF, Horwitz BH, Freeman GJ and Sharpe AH: Regulation of PD-1, PD-L1, and PD-L2 expression during normal and autoimmune responses. Eur J Immunol 33: 2706-2716, 2003.

41. Wang S, Bajorath J, Flies DB, Dong H, Honjo T and Chen L: Molecular modeling and functional mapping of B7-H1 and B7-DC uncouple costimulatory function from PD-1 interaction. J Exp Med 197: 1083-1091, 2003.

42. Elhag OA, Hu XJ, Wen-Ying Z, Li X, Yuan YZ, Deng LF, Liu DL, Liu YL and Hui G: Reconstructed adeno-associated virus with the extracellular domain of murine PD-1 induces antitumor immunity. Asian Pac J Cancer Prev 13: 4031-4036, 2012.

43. Intlekofer AM and Thompson CB: At the bench: Preclinical rationale for CTLA-4 and PD-1 blockade as cancer immunotherapy. J Leukoc Biol 94: 25-39, 2013.

44. Taube JM, Klein A, Brahmer JR, Xu H, Pan X, Kim JH, Chen L, Pardoll DM, Topalian SL and Anders RA: Association of PD-1, PD-1 ligands, and other features of the tumor immune microenvironment with response to anti-PD-1 therapy. Clin Cancer Res 20: 5064-5074, 2014

45. Benson AB, 3rd, D'Angelica MI, Abbott DE, et al: NCCN Guidelines Insights: Hepatobiliary Cancers, Version 1.2017. J Natl Compr Canc Netw 15: 563-573, 2017.

46. El-Khoueiry AB, Sangro B, Yau T, Crocenzi TS, Kudo M, Hsu C, Kim TY, Choo SP, Trojan J, Welling TH Rd, et al: Nivolumab in patients with advanced hepatocellular carcinoma (CheckMate 040): An open-label, non-comparative, phase $1 / 2$ dose escalation and expansion trial. Lancet 389: 2492-2502, 2017.

47. Yau T, Hsu C, Kim TY, Choo SP, Kang YK, Hou MM, Numata K Yeo W, Chopra A, Ikeda M, et al: Nivolumab in advanced hepatocellular carcinoma: Sorafenib-experienced Asian cohort analysis. J Hepatol 71: 543-552, 2019.

48. Sangro B, Park JW, Cruz CMD, Anderson J, Lang L, Neely J, Shaw JW and Cheng AL: A randomized, multicenter, phase 3 study of nivolumab vs sorafenib as first-line treatment in patients (pts) with advanced hepatocellular carcinoma (HCC): CheckMate-459. J Clin Oncol 34 (15 Suppl): TPS4147, 2016.

49. Exposito MJ, Akce M, Alvarez J, Assenat E, Balart L, Baron A, Decaens T, Heurgue-Berlot A, Martin A, Paik S, et al: Abstract No. 526 CheckMate-9DX: Phase 3, randomized, double-blind study of adjuvant nivolumab vs placebo for patients with hepatocellular carcinoma (hcc) at high risk of recurrence after curative resection or ablation. J Vasc Interv Radiology 30: S227-S228, 2019.

50. Zhu AX, Finn RS, Edeline J, Cattan S, Ogasawara S, Palmer D, Verslype C, Zagonel V, Fartoux L, Vogel A, et al: Pembrolizumab in patients with advanced hepatocellular carcinoma previously treated with sorafenib (KEYNOTE-224): A non-randomised, open-label phase 2 trial. Lancet Oncol 19: 940-952, 2018.

51. Finn RS, Chan SL, Zhu AX, Knox JJ, Cheng AL, Siegel AB, Bautista O, Kudo M: Phase 3, randomized study of pembrolizumab (pembro) vs best supportive care (BSC) for second-line advanced hepatocellular carcinoma (HCC): KEYNOTE-240. J Clin Oncol 35 (15 Suppl): TPS4143, 2017.

52. Qin SK Ren ZG, Meng ZQ, Chen ZD, Chai XL, Xiong JP, Bai YX, Yang L, Zhu H, Fang WJ, et al: LBA27A randomized multicentered phase II study to evaluate SHR-1210 (PD-1 antibody) in subjects with advanced hepatocellular carcinoma (HCC) who failed or intolerable to prior systemic treatment. Ann Oncol 29 (Suppl 8): mdy424.029, 2018.
53. Qin SK, Ren Z, Meng Z, Chen Z, Chai X, Xiong J, Bai Y, Yang L, Zhu H, Fang W, et al: Camrelizumab in patients with previously treated advanced hepatocellular carcinoma: A multicentre, open-label, parallel-group, randomised, phase 2 trial. Lancet Oncol 21: 571-580, 2020.

54. Xu JM, Zhang Y, Jia R, Wang Y, Liu R, Zhang G, Zhao C, Zhang Y, Zou J and Wang Q: Anti-programmed death-1 antibody SHR-1210 (S) combined with apatinib (A) for advanced hepatocellular carcinoma (HCC), gastric cancer (GC) or esophagogastric junction (EGJ) cancer refractory to standard therapy: A phase 1 trial. J Clin Oncol 36 (15 Suppl): S4075, 2018.

55. Qin S, Chen Z, Liu Y, Xiong J, Ren Z, Meng Z, Gu S, Wang L, Zou J; Jinling Hospital, et al: A phase II study of anti-PD-1 antibody camrelizumab plus FOLFOX4 or GEMOX systemic chemotherapy as first-line therapy for advanced hepatocellular carcinoma or biliary tract cancer. J Clin Oncol 37 (15 Suppl): S4074, 2019.

56. Pishvaian MJ, Lee M, Ryoo BY, Stein S, Lee KH, Stein W, Spahn J, Shao H, Liu B and Iizuka K: LBA26Updated safety and clinical activity results from a phase Ib study of atezolizumab + bevacizumab in hepatocellular carcinoma (HCC). Ann Oncol 29 (Suppl 8): viii718-viii719, 2018.

57. Cheng AL, Qin S, Ikeda M, Galle P, Ducreux M, Zhu A, Kim TY, Merle P, Kaseb A, Li D, et al: IMbrave150: Efficacy and safety results from a ph III study evaluating atezolizumab (atezo)+ bevacizumab (bev) vs sorafenib (Sor) as first treatment (tx) for patients (pts) with unresectable hepatocellular carcinoma (HCC). Ann Oncol 30: ix186-ix187, 2019.

58. Qin SK, Ren ZG, Feng Y, et al: Efficacy and safety of atezolizumab + bevacizumab vs sorafenib in Chinese patients with unresectable HCC in the phase III IMbrave150 study. EASL Liver Cancer Summit 2020. OP02-03. Ann Oncol 30: v875, 2020.

59. Kudo M, Finn RS, Qin S, Han KH, Ikeda K, Piscaglia F, Baron A, Park JW, Han G, Jassem J, et al: Lenvatinib versus sorafenib in first-line treatment of patients with unresectable hepatocellular carcinoma: A randomised phase 3 non-inferiority trial. Lancet 391: 1163-1173, 2018.

60. Ikeda M, Sung MW, Kudo M, Kobayashi M, Baron AD, Finn RS, Kaneko S, Kraljevic S, Ishikawa K, Siegel AB, et al: A phase $1 \mathrm{~b}$ trial of lenvatinib (LEN) plus pembrolizumab (PEM) in patients (pts) with unresectable hepatocellular carcinoma (uHCC). J Clin Oncol 36 (15 Suppl): S4076, 2018.

61. Jeon MY, Lee HW, Kim BK, Park JY, Kim DY, Ahn SH, Han KH, Baek SE, Kim HS, Kim SU and Park MS: Reproducibility of European Association for the Study of the liver criteria and modified response evaluation criteria in solid tumors in patients treated with sorafenib. Liver Int 38: 1655-1663, 2018

62. Llovet J, Shepard KV, Finn RS, Ikeda M, Sung M, Baron DA, Kudo M, Okusaka T, Kobayashi M, Kumada H, et al: A phase Ib trial of lenvatinib (LEN) plus pembrolizumab (PEMBRO) in unresectable hepatocellular carcinoma (uHCC): Updated results. Ann Oncol 30: v286-v287, 2019.

63. Kudo M, Ikeda M, Motomura K, Okusaka T, Kato N, Dutcus CE Hisai T, Suzuki M, Ikezawa H, Iwata T, et al: A phase $1 \mathrm{~b}$ study of lenvatinib plus nivolumab in patients with unresectable hepatocellular carcinoma (Study 117). J Clin Oncol 38 (4 Suppl): S513, 2020.

64. Agdashian D, ElGindi M, Xie C, Sandhu M, Pratt D, Kleiner DE, Figg WD, Rytlewski JA, Sanders C, Yusko EC, et al: The effect of anti-CTLA4 treatment on peripheral and intra-tumoral T cells in patients with hepatocellular carcinoma. Cancer Immunol Immunother 68: 599-608, 2019.

65. Sangro B, Gomez-Martin C, de la Mata M, Iñarrairaegui M, Garralda E, Barrera P, Riezu-Boj JI, Larrea E, Alfaro C, Sarobe P, et al: A clinical trial of CTLA-4 blockade with tremelimumab in patients with hepatocellular carcinoma and chronic hepatitis C. J Hepatol 59: 81-88, 2013.

66. Yau T, Kang YK, , Kim TY, El-Khoueiry AB, Santoro A, Sangro B, Melero I, Kudo M, Hou MM, Matilla A, et al: Nivolumab (NIVO) + ipilimumab (IPI) combination therapy in patients (pts) with advanced hepatocellular carcinoma (aHCC): Results from CheckMate 040. J Clin Oncol 37 (15 Suppl): S4012, 2019.

67. Finkelmeier F, Waidmann O and Trojan J: Nivolumab for the treatment of hepatocellular carcinoma. Expert Rev Anticancer Ther 18: 1169-1175, 2018.

68. Opdivo Prescribing Information. Opdivo U.S.Product Information.Last updated: March 2020. Princeton, NJ: Bristol Myers Squibb Company.

69. Abou-Alfa GK, Chan SL, Furuse J, Galle PR, Kelley RK, Qin S, Armstrong J, Darilay A, Vlahovic G, Negro A and Sangro B: A randomized, multicenter phase 3 study of durvalumab (D) and tremelimumab $(\mathrm{T})$ as first-line treatment in patients with unresectable hepatocellular carcinoma (HCC): HIMALAYA study. J Clin Oncol 36 (15 Suppl): TPS4144, 2018 
70. Kelley RK , Abou-Alfa GK, Bendell JC, Kim TY, Borad MJ, Yong WP, Morse M, Kang YK, Rebelatto M, Makowsky M, et al: Phase I/II study of durvalumab and tremelimumab in patients with unresectable hepatocellular carcinoma (HCC): Phase I safety and efficacy analyses. J Clin Oncol 35 (15 Suppl): S4073, 2017.

71. Choi C, Yoo GS, Cho WK and Park HC: Optimizing radiotherapy with immune checkpoint blockade in hepatocellular carcinoma. World J Gastroenterol 25: 2416-2429, 2019

72. Hilmi M, Neuzillet C, Calderaro J, Lafdil F, Pawlotsky JM and Rousseau B: Angiogenesis and immune checkpoint inhibitors as therapies for hepatocellular carcinoma: current knowledge and future research directions. J Immunother Cancer 7: 333, 2019.

73. Xu W, Liu K, Chen M, Sun JY, McCaughan GW, Lu XJ and Ji J: Immunotherapy for hepatocellular carcinoma: Recent advances and future perspectives. Ther Adv Med Oncol 11: $1758835919862692,2019$.

74. Loosen SH, Schulze-Hagen M, Bruners P, Tacke F, Trautwein C, Kuhl C, Luedde T and Roderburg C: Sarcopenia is a negative prognostic factor in patients undergoing transarterial chemoembolization (TACE) for hepatic malignancies. Cancers (Basel) 11: 1503, 2019.

75. Harding JJ, Erinjeri JP, Tan BR, Reiss KA, Mody K, Khalil D, Yarmohammadi H, Nadolski G, Giardina JD, Capanu M, et al: A multicenter pilot study of nivolumab (NIVO) with drug eluting bead transarterial chemoembolization (deb-TACE) in patients (pts) with liver limited hepatocellular carcinoma (HCC). J Clin Oncol 36 (15 Suppl): TPS4146, 2018.

76. Chen CL, Pan QZ, Zhao JJ, Wang Y, Li YQ, Wang QJ, Pan K, Weng DS, Jiang SS, Tang Y, et al: PD-L1 expression as a predictive biomarker for cytokine-induced killer cell immunotherapy in patients with hepatocellular carcinoma. Oncoimmunology 5: e1176653, 2016.

77. Zhu AX, Kang YK, Yen CJ, Finn RS, Galle PR, Llovet JM, Assenat E, Brandi G, Pracht M,Lim HY, et al: Ramucirumab after sorafenib in patients with advanced hepatocellular carcinoma and increased $\alpha$-fetoprotein concentrations (REACH-2): A randomised, double-blind, placebo-controlled, phase 3 trial. Lancet Oncol 20: 282-296, 2019.

78. Zhu AX, Park JO, Ryoo BY, Yen CJ, Poon R, Pastorelli D, Blanc JF, Chung HC, Baron AD, Pfiffer TE, et al: Ramucirumab versus placebo as second-line treatment in patients with advanced hepatocellular carcinoma following first-line therapy with sorafenib $(\mathrm{REACH})$ : A randomised, double-blind, multicentre, phase 3 trial. Lancet Oncol 16: 859-870, 2015.

79. Sideras K, Biermann K, Verheij J, Takkenberg BR, Mancham S, Hansen BE, Schutz HM, de Man RA, Sprengers D, Buschow SI, et al: PD-L1, Galectin-9 and CD8+ tumor-infiltrating lymphocytes are associated with survival in hepatocellular carcinoma. Oncoimmunology 6: e1273309, 2017

80. Li H, Wu K, Tao K, Chen L, Zheng Q, Lu X, Liu J, Shi L, Liu C, Wang G and Zou W: Tim-3/galectin-9 signaling pathway mediates T-cell dysfunction and predicts poor prognosis in patients with hepatitis B virus-associated hepatocellular carcinoma. Hepatology 56: 1342-1351, 2012.
81. Eso Y, Shimizu T, Takeda H, Takai A and Marusawa H: Microsatellite instability and immune checkpoint inhibitors: Toward precision medicine against gastrointestinal and hepatobiliary cancers. J Gastroenterol 55: 15-26, 2020.

82. Latham A, Srinivasan P, Kemel Y, Shia J, Bandlamudi C, Mandelker D, Middha S, Hechtman J, Zehir A, Dubard-Gault M, et al: Microsatellite instability is associated with the presence of lynch syndrome pan-cancer. J Clin Oncol 37: 286-295, 2019.

83. Le DT, Durham JN, Smith KN, Wang H, Bartlett BR, Aulakh LK, Lu S, Kemberling H, Wilt C, Luber BS, et al: Mismatch repair deficiency predicts response of solid tumors to PD-1 blockade. Science 357: 409-413, 2017.

84. Kawaoka T, Ando Y, Yamauchi M, Suehiro Y, Yamaoka K, Kosaka Y, Fuji Y, Uchikawa S, Morio K, Fujino H, et al: Incidence of microsatellite instability-high hepatocellular carcinoma among Japanese patients and response to pembrolizumab. Hepatol Res 50: 885-888, 2020.

85. Goumard C, Desbois-Mouthon C, Wendum D, Calmel C, Merabtene F, Scatton O and Praz F: Low levels of microsatellite instability at simple repeated sequences commonly occur in human hepatocellular carcinoma. Cancer Genomics Proteomics 14: 329-339, 2017.

86. Ando Y, Yamauchi M, Suehiro Y, Yamaoka K, Kosaka Y, Fuji Y, Uchikawa S, Kodama K, Morio K, Fujino H, et al: Complete response to pembrolizumab in advanced hepatocellular carcinoma with microsatellite instability. Clin J Gastroenterol, Feb 4, 2020 (Online ahead of print).

87. Scheiner B, Kirstein MM, Hucke F, Finkelmeier F, Schulze K, von Felden J, Koch S, Schwabl P, Hinrichs JB, Waneck F, et al: Programmed cell death protein-1 (PD-1)-targeted immunotherapy in advanced hepatocellular carcinoma: Efficacy and safety data from an international multicentre real-world cohort. Aliment Pharmacol Ther 49: 1323-1333, 2019.

88. Chen W, Zheng R, Baade PD, Zhang S, Zeng H, Bray F, Jemal A, $\mathrm{Yu}$ XQ and He J: Cancer statistics in China, 2015. CA Cancer J Clin 66: 115-132, 2016.

89. Boni C, Barili V, Acerbi G, Rossi M, Vecchi A, Laccabue D, Penna A, Missale G, Ferrari C and Fisicaro P: HBV immune-therapy: from molecular mechanisms to clinical applications. Int J Mol Sci 20: 2754, 2019.

90. Gao L, Yang X, Yi C and Zhu H: Adverse events of concurrent immune checkpoint inhibitors and antiangiogenic agents: A systematic review. Front Pharmacol 10: 1173, 2019.

This work is licensed under a Creative Commons Attribution-NonCommercial-NoDerivatives 4.0 International (CC BY-NC-ND 4.0) License. 